\title{
Onde ou particule, le photon défie toujours l'intuition
}

\author{
Sébastien TANZILLI', Florian KAISER', Thomas COUDREAU ${ }^{2}$, Pérola MILMAN², Daniel B. OSTROWSKY' \\ ' Laboratoire de Physique de la Matière Condensée, CNRS UMR 7336, \\ Université Nice Sophia Antipolis, 06108 Nice Cedex 2, France \\ ${ }^{2}$ Laboratoire Matériaux et Phénomènes Quantique, CNRS UMR 7162, \\ Université Paris Diderot, Sorbonne Paris Cité, 75013 Paris, France \\ sebastien.tanzilli@unice.fr
}

La dualité onde/particule se teste la plupart du temps à l'aide de photons uniques envoyés dans un interféromètre de Mach-Zehnder équilibré. On peut alors observer des comportements considérés comme mutuellement exclusifs, à savoir ondulatoire (présence d'interférences) ou corpusculaire (absence d'interférences) selon la présence ou l'absence de la lame séparatrice en sortie de l'interféromètre.

Nous présentons un nouveau type d'expérience de complémentarité dans laquelle cette lame séparatrice est préparée dans une superposition cohérente lui permettant d'être à la fois présente et absente. Cette préparation est « activée » grâce à l'emploi de paires de photons intriqués en polarisation. L'un des photons, dit test, est envoyé dans l'interféromètre et est détecté alors que son jumeau, dit corroborant, permet de déterminer si un comportement de type ondulatoire, corpusculaire ou intermédiaire a été observé pour le photon test. En manipulant l'état de polarisation du photon corroborant et la phase de l'interféromètre du photon test, nous observons une transition continue entre aspects ondulatoire et corpusculaire, signature forte de la notion de complémentarité introduite par Bohr et donc de la non exclusivité de ces comportements.

\section{La (petite) histoire des interprétations données à la lumière}

Les Hommes depuis l'antiquité se sont intéressés aux effets liés à la lumière et notamment à la genèse des couleurs qui, à travers un phénomène comme l'arc-enciel, leur apparaissaient de façon spectaculaire. Pour Aristote (384-322 av. J.-C.), l'apparition des couleurs était liée à un affaiblissement de la lumière blanche, une idée qui n'évoluera guère pendant près de deux mille ans. En 1665, Robert Hooke observa les couleurs de lames minces de mica. Pour décrire ce phénomène, il proposa, dans le cadre de sa théorie ondulatoire de la lumière, de combiner deux rayons lumineux, approche qui préfigure la théorie moderne (classique) des interférences, mais qui, malheureusement, restera qualitative. II fut suivi de près par Christiaan Huygens qui expliqua en 1690 le phénomène de diffraction, toujours en se fondant sur une théorie ondulatoire. Dans le même temps, Isaac Newton en 1675, dans le cadre de sa théorie corpusculaire de la lumière, expliqua quantitativement l'observation, dans une lame d'air d'épaisseur variable, d'un système d'anneaux qui porte son nom. Sa notoriété scientifique imposa son point de vue et, malgré les travaux de Hooke et Huygens, la description ondulatoire attendra plus d'un siècle avant de revenir sur le devant de la scène. En 1801, Thomas Young réalisa en effet sa fameuse expérience d'interférences à l'aide d'un dispositif expérimental composé d'une double fente et observa une figure d'interférences, signature non ambigüe d'un phénomène ondulatoire. Par la suite, Augustin Fresnel expliqua la polarisation de la lumière et ses effets dans les cristaux biréfringents. En 1865, James C. Maxwell jeta enfin les bases d'une théorie ondulatoire complète, unifiant champs électrique et magnétique, théorie que l'on connaît aujourd'hui sous le nom d'électromagnétisme et qui se résume à quatre fameuses équations. À la fin du XIXe siècle, beaucoup pensaient que l'histoire se terminerait là, mais c'était sans compter sur l'avènement de la physique quantique. Dès 1900, Max Planck résolut enfin le problème du corps noir en quantifiant les échanges d'énergie entre les modes du champ électromagnétique et le réservoir dans lequel il évolue. Un quantum d'énergie électromagnétique est proportionnel à la fréquence du champ qui lui est associé, à un tout petit facteur « $\mathrm{h}$ » près ... la constante de Planck. L'ère de la « lumière quantique » et du concept de « photon » pouvait enfin commencer.

Planckfut suivi de près par AlbertEinstein qui explicita l'effet photoélectrique selon 
le même principe de quantification des échanges d'énergie. II montra en effet qu'un métal peut libérer un électron lorsque la fréquence du faisceau lumineux incident correspond à son potentiel chimique de libération. En 1927, Paul A. M. Dirac parlait déjà d'interférence «à photons uniques » en sortie d'un dispositif de type interféromètre de Mach-Zehnder équilibré. Pour Dirac, les photons dans l'interféromètre se trouvent dans un état «translationnel», autrement dit dans une superposition cohérente entre les deux bras du dispositif (voir encadré), affirmant de plus que chaque photon interfère avec lui-même.

Alors le photon, la lumière, ondes ou particules?

Essayons en tout cas, et espérons-le, d'y voir un peu plus clair dans ce qui suit..

\section{Observation de comportements complémentaires et exclusifs}

La notion de complémentarité onde/ particule de Bohr est au cœur de la physique quantique. Interprétée de façon naïve en terme de dualité, elle suggère que les systèmes quantiques individuels se comportent comme des ondes ou des particules suivant le type de mesure qui leur est appliqué, autrement dit selon l'arrangement expérimental auquel ils sont soumis [1].
Comme le montre le dispositif expérimental de la figure $1 a$, lorsque des photons uniques sont envoyés dans un interféromètre de Mach-Zehnder (MZI) fermé ou ouvert, ou, en d'autres termes, lorsque la lame séparatrice de sortie $\left(\mathrm{BS}_{\text {out }}\right.$ pour «beam-splitter » de sortie) est présente ou absente, nous observons respectivement un comportement ondulatoire (figure d'interférences, figure 1b) ou corpusculaire (pas d'interférences, figure 1c). Ces comportements mutuellement exclusifs ont été observés pour la première fois à l'aide d'une source de photons uniques par P. Grangier et ses collaborateurs en 1986 [2].

\section{Expérience à choix retardé}

Àla même époque, Wheeler proposa en 1984 une expérience de pensée destinée à réfuter les modèles à variables cachées supposés fournir aux photons une indication sur la configuration expérimentale (MZI fermé ou ouvert) à laquelle ils vont être soumis, configuration qui est généralement fixée avant que l'expérience ne débute réellement [3]. Pour trancher ce débat, il convient donc d'effectuer un test à choix retardé permettant à l'expérimentateur de configurer l'interféromètre après que le photon a déjà franchi la lame séparatrice d'entrée $\left(\mathrm{BS}_{\text {in }}\right.$ à la figure $\left.1 a\right)$ et qu'il se trouve, par conséquent, déjà « quelque part » dans le dispositif.
Cette expérience, réalisée en 2007 parV. Jacques et ses collaborateurs à l'aide d'une source déterministe de photons uniques, a montré que la notion de complémentarité de Bohr était toujours respectée [4]. II a également été conclu que l'instant du choix de la configuration expérimentale de l'interféromètre ne semble jouer aucun rôle dans cette affaire.

\section{Expérience quantique à choix (très) retardé}

Il a été récemment proposé de franchir une étape supplémentaire dans l'expérience de Wheeler en employant une "lame séparatrice de sortie quantique » (QBS, quantum beam-splitter), ou, en d'autres termes, une lame préparée dans une superposition cohérente d'états lui permettant d'être à la fois présente et absente. L'état de la lame séparatrice quantique peut alors s'écrire sous la forme $|\mathrm{QBS}\rangle=\mu\left|\mathrm{BS}_{\text {out }}^{p}\right\rangle+\xi\left|\mathrm{BS}_{\text {out }}^{a}\right\rangle$ (équation 1) où $p$ et $a$ indiquent respectivement présente et absente et où $\mu$ et $\xi$ sont les poids de la superposition [5].

Comme l'indique la figure 2 , nous avons réalisé cette expérience en exploitant deux ressources, d'une part des paires de photons intriqués en polarisation et, d'autre part, une lame séparatrice particulière dont le rôle est décrit plus bas.

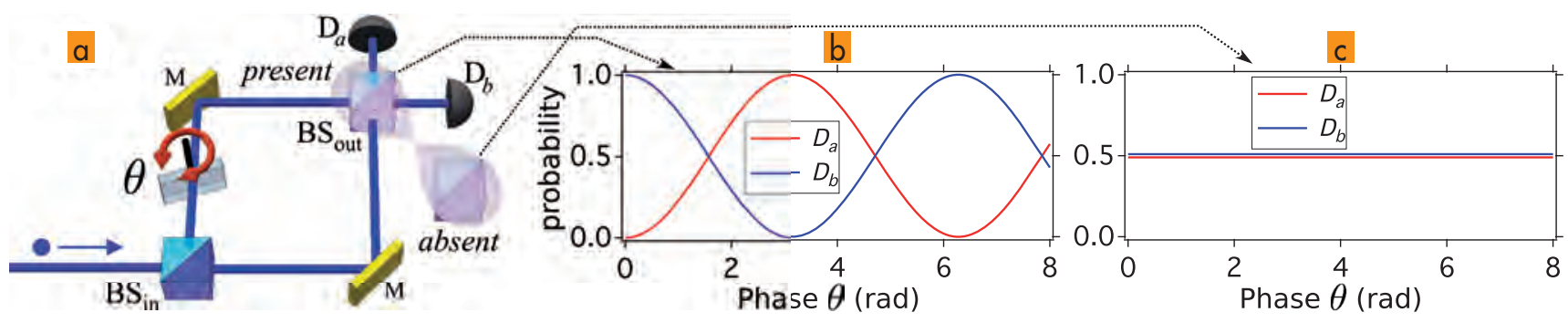

Figure 1. (a) Dispositif expérimental basé sur un MZI équilibré permettant d'observer la dualité onde/corpuscule exclusive. Lorsque la lame séparatrice de sortie $\left(\mathrm{BS}_{\text {out }}\right.$ ) est présente, on observe des oscillations (interférences), signature d'un phénomène ondulatoire, voir courbe (b) [2]. Dans cette configuration, les probabilités de détecter un photon dans l'un ou l'autre des détecteurs oscillent en opposition de phase $(\theta)$, à mesure que l'expérimentateur fait varier la phase dans l'un des bras de l'interféromètre. II n'est par ailleurs pas possible de déterminer par quel chemin le photon est passé dans le dispositif interférométrique. De façon complémentaire, orsque $B S_{\text {out }}$ est absente, un clic dans le détecteur $D_{a}$ ou $D_{b}$ réduit la superposition cohérente en chemin créée au niveau de la lame séparatrice d'entrée ( $B S_{\text {in }}$ ) à l'une des deux possibilités, à savoir chemin du haut ou chemin du bas. Dans le cas où le signal lumineux employé est de type photons uniques, cette configuration es souvent admise comme une façon de révéler le comportement corpusculaire des photons, voir courbe (c). Notons enfin que si la lame d'entrée est de type 50/50, la probabilité de détecter un photon dans la configuration ouverte vaut alors $1 / 2$ quel que soit le détecteur considéré.

Notations: $\mathrm{BS}_{\text {in }}$ et $\mathrm{BS}_{\text {out' }}$ respectivement lame séparatrice d'entrée et de sortie de l'interféromètre de Mach-Zehnder $(M Z I) ; \theta$, phase de l'interféromètre ; $D_{a}$ et $D_{b^{\prime}}$ détecteurs placés en sortie de l'interféromètre. 
Tout d'abord, les paires de photons sont préparées dans l'état

$\left|\Psi_{c, t}\right\rangle=1 / \sqrt{ } 2\left[\left|H_{c}\right\rangle\left|H_{t}\right\rangle+\left|V_{c}\right\rangle\left|V_{t}\right\rangle\right]$

(équation 2)

où $\mathrm{H}$ et $\mathrm{V}$ représentent respectivement les états de polarisation horizontal et vertical des photons [6] (voir également l'encadré).

L'un des photons, dit test $(t)$, est alors envoyé dans un MZI dont la configuration (ouvert/fermé) peut être manipulée de façon cohérente au travers de l'état intriqué via la mesure de l'état de polarisation du second photon, dit corroborant (c). C'est la première brique nécessaire à l'implémentation du QBS.

Par ailleurs, sachant que l'état intriqué (équation 2) est non séparable (voir encadré), celui-ci garantit que l'état de polarisation des photons test est indéfini. Nous avons donc conçu une lame séparatrice en sortie du MZI dont l'effet dépend de l'état de polarisation (PDBS à la figure 2, pour polarization dependent beam-splitter). En effet, elle est de type 50/50 pour la composante $\mathrm{V}$ de polarisation, ce qui équivaut à la présence d'une lame séparatrice standard (voir figure 1), mais est $100 \%$ réfléchissante pour la composante $\mathrm{H}$, ce qui équivaut à l'absence de la lame. Ceci constitue la seconde brique nécessaire à l'implémentation du QBS qui offre donc la possibilité aux photons test de se comporter à la fois comme une onde ou comme des particules.

L'implémentation « quantique et matérielle » du QBS permet par conséquent

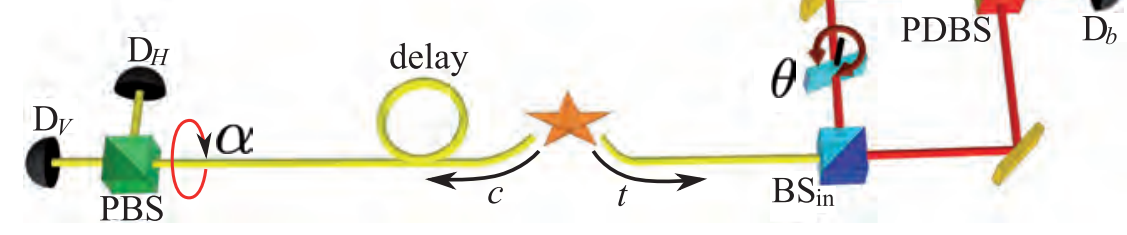

Figure 2. Expérience quantique à choix retardé activée par l'intrication. D'une part, une source délivre des paires de photons intriqués en polarisation préparées dans l'état donné par l'équation 2 [6]. D'autre part, par rapport à la figure 1, la lame séparatrice de sortie est « physiquement » remplacée par une lame dont l'effet dépend de l'état de polarisation qui lui est envoyé (PDBS, voir texte) et qui offre aux photons test la possibilité de se comporter à la fois comme une onde (composante $V$ de polarisation), ou comme des particules (composante H). C'est bien l'association du PDBS et de l'intrication qui autorise la manipulation cohérente d'une lame séparatrice quantique (QBS) et l'observation de superpositions de comportements pour les photons test [7].

Notations : $c$ et $t$, paire de photons test ( $t$ ) et corroborant $(c)$, intriqués en polarisation; PBS, cube polarisant (polarization beam splitter) ; $D_{V}$ et $D_{H^{\prime}}$ détecteurs mesurant respectivement l'aspect ondulatoire et l'aspect corpusculaire ; $\alpha$, angle de rotation du cube polarisant (PBS) permettant d'analyser l'état de polarisation du photon corroborant dans une base d'états de polarisation non alignée avec la base d'origine (c); $\mathrm{BS}_{i n^{\prime}}$ lame séparatrice d'entrée de l'interféromètre de Mach-Zehnder ; $\theta$, phase de l'interféromètre ; PDBS, cube séparateur placé en sortie du MZI et dont l'effet dépend de l'état de polarisation (polarization dependent beam splitter) ; $D_{a}$ et $D_{b}$, détecteurs placés en sortie de l'interféromètre.

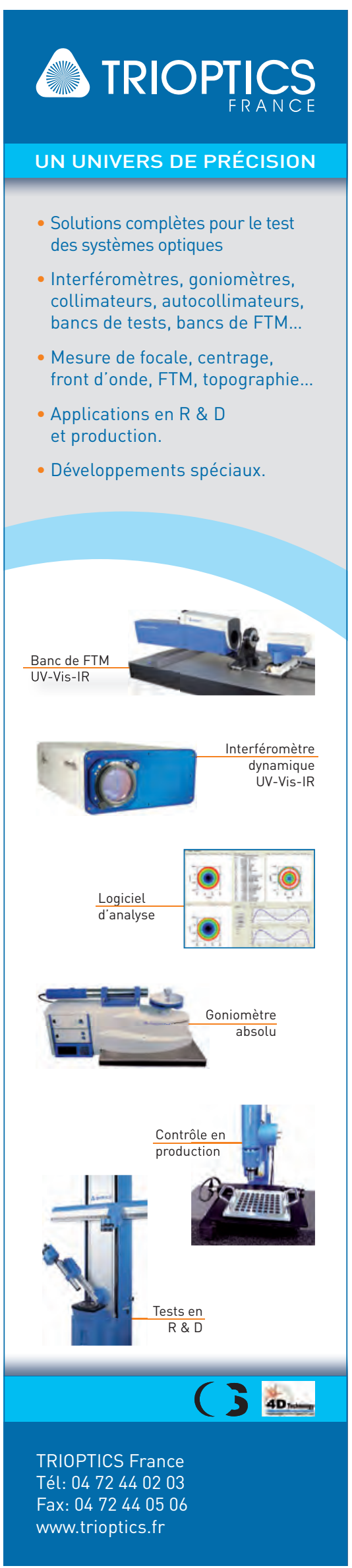




\section{Superposition cohérente d'état et intrication phołonique en polarisction}

\section{La superposition cohérente d'états}

Envoyer un photon sur une lame séparatrice (comme par exemple $\mathrm{BS}_{\text {in }}$ à la figure 1) revient à la préparer dans une superposition cohérente sur l'observable " chemin optique » relative aux deux voies de sortie. Si la lame séparatrice est de type 50/50, l'état du photon peut être écrit comme $1 / \sqrt{ } 2$ (i|refle $\rangle+\mid$ trans $\rangle)$ où « refle » et « trans » représentent les voies de réflexion et de transmission. C'est ce que Dirac appelait un état " translationnel». Afin d'expliciter par la suite l'état intriqué en polarisation de l'équation 2 , considérons à présent l'observable polarisation du photon.

Nous savons aujourd'hui produire des photons uniques préparés dans un état de polarisation horizontal, ou vertical, ce qui s'écrit respectivement $|\Psi\rangle=|H\rangle$ ou $|\Psi\rangle=|V\rangle$.

Ces états de base peuvent être obtenus en plaçant simplement une lame demi-onde en sortie d'une source émettant des photons uniques

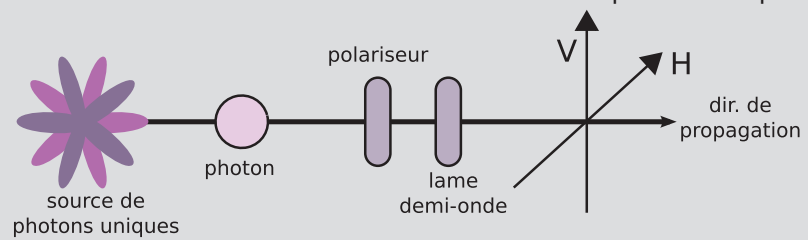

\section{L'intrication photonique en polarisation}

L'intrication correspond à la généralisation à deux ou plusieurs systèmes quantiques de la superposition cohérente d'états définie ci-dessus.

Par exemple, la figure 5 représente une source émettant des paires de photons intriqués sur l'observable polarisation, dont l'état est donné par l'équation 2. Rappelons que les labels $c$ et treprésentent dans notre cas le photon corroborant et le photon test.

Du point de vue du formalisme quantique, cet état est dit intriqué car il n'est, mathématiquement, pas factorisable selon ses constituants. Autrement dit, il est rigoureusement impossible d'écrire $\left|\Psi_{c, t}\right\rangle=\left|\Psi_{c}\right\rangle\left|\Psi_{t}\right\rangle$, ce qui correspondrait à la description d'un état séparable ! Par conséquent, en ce qui concerne l'observable polarisation, seul l'état quantique de la paire de photons est défini alors que les états individuels des photons $c$ et $t$ ne le sont pas. Physiquement, une paire de photons intriqués doit

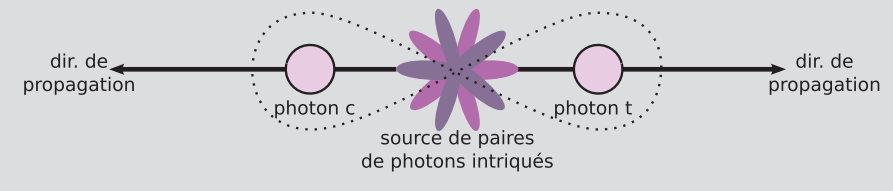

polarisés linéairement (figure 4). C'est alors l'angle avec lequel la lame est orientée par l'expérimentateur dans le plan perpendiculaire à la direction de propagation qui permet de définir l'état de polarisation codé sur le photon considéré. Toutefois, la physique quantique permet également, en configurant la lame demi-onde selon un angle approprié, de préparer les photons dans une superposition cohérente définie dans la base de polarisation $\{|H\rangle,|V\rangle\}$ par $\mu|\mathrm{H}\rangle+\xi|\mathrm{V}\rangle$ (équation 4)

où $\mu=\cos (\delta)$ et $\xi=\sin (\delta)$ sont les amplitudes de probabilités associées aux deux états de base et où $\delta$ représente le double de l'angle avec lequel la lame demi-onde est configurée. On voit que $\mu$ et $\xi$ vérifient, quel que soit $\delta$, la règle de normalisation $|\mu|^{2}+|\xi|^{2}=1$. Par exemple, l'angle $\delta=45^{\circ}$, correspondant à une lame demionde orientée à $22,5^{\circ}$, permet de préparer l'état diagonal $|\mathrm{D}\rangle=1 / \sqrt{ } 2|| \mathrm{H}\rangle+|\mathrm{V}\rangle\rangle$.

Figure 4. Une source émet des photons uniques. Le polariseur leur affecte un état de polarisation linéaire qui peut être contrôlé en orientant la lame demi-onde de façon appropriée. Faire tourner la lame d'un angle donné revient à jouer sur les amplitudes de probabilités donnés aux états de base $|H\rangle$ et $|V\rangle$ (voir équation 4). est déterminé via une mesure de l'état de polarisation du photon c seulement après la détection du photon test. L'intervalle du genre espace séparant les mesures respectives du photon $t$ et du photon $c$ invalide les modèles à variables cachées locales associés à une information préexistante sur les résultats des mesures. Nous vérifions notamment la qualité de l'intrication par la violation des inégalités de Bell avec plus de 10 déviations donc être considérée comme un tout, c'est-à-dire un système quantique unique composé de deux sous-systèmes et ce, même si les photons sont séparés spatialement.

Précisons que la méthode la plus couramment employée pour générer des paires de photons intriqués en polarisation consiste à faire interagir un laser de pompe avec un cristal non-linéaire d'ordre 2, les paires de photons étant émises via le processus bien connu de conversion paramétrique. Un bon exemple de source de paires de photons intriqués en polarisation est donné en [6].

Notons enfin que la superposition cohérente d'états et plus particulièrement l'intrication sont longtemps restées des concepts fondamentaux. Aujourd'hui ces états servent de ressources aux protocoles de communication et de traitement quantique de l'information. Le protocole le plus connu est sans doute l'établissement quantique de clés de cryptographie entre partenaires distants, communément nommé « cryptographie quantique ».

Figure 5. Une source émet des paires de photons intriqués préparées dans l'état donné par l'équation 2. La paire de photons doit être considérée comme un tout, c'est-à-dire comme un système quantique unique, depuis son instant de création jusqu'aux instants où les photons sont détectés.

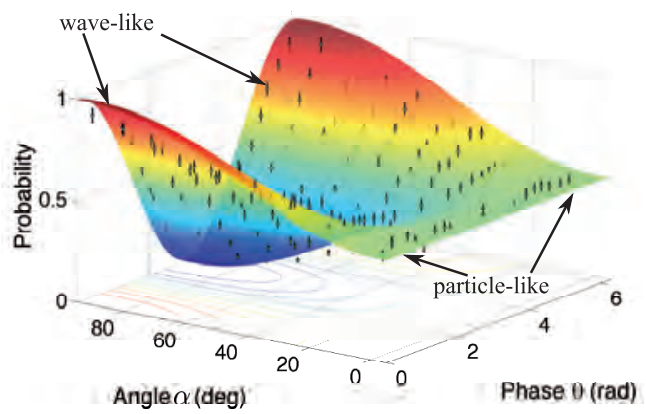

standards. En d'autres termes, lorsque le photon test est détecté, aucune information sur la mesure qui lui a été appliquée n'existe dans la nature. Notons enfin que

Figure 3. Transition continue entre comportements ondulatoire et corpusculaire via la manipulation du QBS en sortie du MZI (voir figure 2) et la phase ajustable $\theta$ placée dans l'un de ses bras. $\alpha$ correspond à l'angle d'analyse de l'état de polarisation du photon corroborant. 
ces résultats sont en parfait accord avec la notion de complémentarité de Bohr [1] et sa généralisation [8].

\section{Des résultats en parfait accord avec la physique quantique}

L'intrication est au cœur de l'approche présentée ci-dessus. Elle permet en effet d'observer des photons uniques selon des aspects purement ondulatoire, purement corpusculaire, mais également dans toutes les superpositions d'états intermédiaires grâce à l'activation et à la manipulation cohérente d'une lame séparatrice quantique en sortie de l'interféromètre de Mach-Zehnder.

Les résultats de cette version quantique de l'expérience de Wheeler ne peuvent être expliqués en termes classiques, ou alors au prix de sévères contradictions. En effet, il est toujours tentant d'essayer d'expliquer la dualité onde/corpuscule prise dans sa version simple par la connaissance ou non du chemin emprunté par les photons dans l'interféromètre, selon qu'ils se comportent respectivement comme des particules ou comme une onde. En revanche, ces résultats sont en parfait accord avec la physique quantique pour laquelle le chemin emprunté par les photons dans l'interféromètre ne représente pas une question pertinente.

Par ailleurs, il convient de noter que le temps, ou plus spécifiquement les instants relatifs auxquels les mesures tet $c$ sont effectuées, et l'espace, ou plus spécifiquement dans notre cas le maintien de l'intrication au-delà d'un intervalle du genre espace, ne semblent jouer aucun rôle [7]. Concernant l'absence de rôle joué par le temps, certains parlent de «procrastination quantique», ou comment reporter à demain ce que l'on est censé faire aujourd'hui...

\section{Remerciements}

M. Le Bellac et J.-P. Romagnan sont remerciés pour leurs suggestions éclairées.

\section{Références}

[1] N. Bohr, "The quantum postulate and the recent development of atomic theory ", Nature 121, pp. 580-590 (1928).

[2] P. Grangier et al., «Experimental evidence for a photon anticorrelation effect on a beam splitter: a new light on single-photon interferences », Europhys. Lett. 1, pp. 173-179 (1986).

[3] J.A. Wheeler in Quantum Theory \& Measurement, J.A. Wheeler \& W.H. Zurek Eds. (Princeton Univ. Press, NJ, 1984), pp. 182-213.

[4] V. Jacques et al., "Experimental realization of Wheeler's delayed-choice gedanken experiment », Science 315, pp. $966-968$ (2007).

[5] R. Ionicioiu and D.R. Terno, «Proposal for a quantum delayed-choice experiment », Phys. Rev. Lett. 107, 230406 (2011).

[6] F. Kaiser et al., " $A$ versatile source of polarization entangled photons for quantum network applications », Laser Phys. Lett. 10, 045202 (2013).

[7] F. Kaiser et al., "Entanglement-enabled delayed-choice experiment », Science 338, pp 637-640 (2012), et les 2 références associées incluses dans le même numéro.

[8] B.-G. Englert, Phys. Rev. Lett. 77, pp. 2154 2157 (1996)

\title{
IL EXISTE PEU DE CHOSES
} AUSSI PETITES ET PUISSANTES QUE NOTRE NOUVELLE CAMÉRA XS

\author{
Si petite, si simple, tellement \\ géniale
}

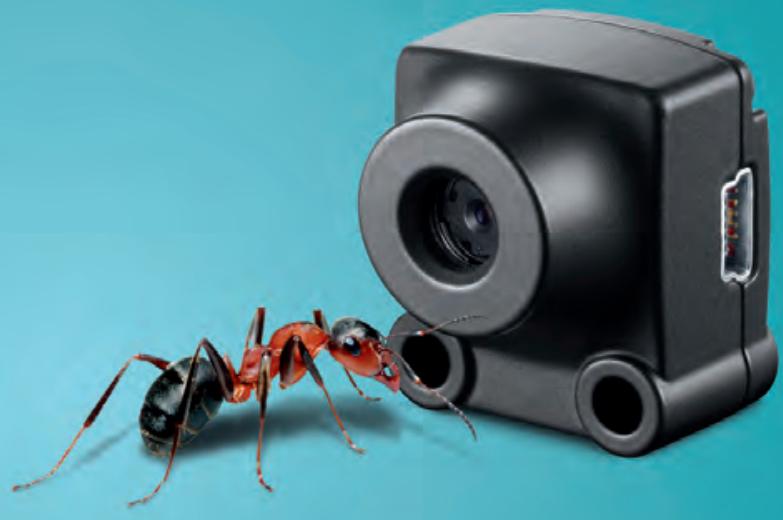

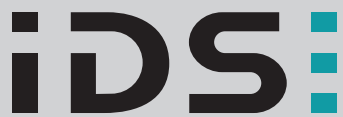

www.ids-imaging.fr/xs
La caméra USB $\mathbf{2 . 0}$ pour toutes les possibilités d'utilisation. Notre minuscule XS

$(23 \times 26,5 \times 21,5 \mathrm{~mm})$ recèle de nombreuses fonctions utiles. Un capteur CMOS moderne avec autofocus, gain auto, exposition automatique, fonction anti-scintillement, calcul des couleurs, réglage de finesse et un zoom numérique garantissent des captures simples dans toutes les conditions de luminosité et tous les environnements. Il suffit de déclencher et voilà ! La longue expérience d'IDS, fabricant leader dans le monde entier de caméras industrielles USB, est la pierre angulaire de cette technologie. La suite logicielle éprouvée IDS est incluse. It’s so easy! 